\title{
Delayed diagnosis of cleidocranial dysplasia in an adult: A case report
}

\author{
Cengiz Gömleksiz', Erhan Arslan², Selçuk Arslan ${ }^{3}$, Serhat Pusat ${ }^{4}$, Elif Acar Arslan ${ }^{5}$ \\ ${ }^{1}$ Department of Neurosurgery \\ Mengucekgazi Training and Research \\ Hospital, Erzincan University \\ Erzincan Turkey \\ ${ }^{2}$ Department of Neurosurgery \\ Giresun University, School of Medicine \\ Giresun, Turkey \\ ${ }^{3}$ Department of Otorhinolaryngology \\ Kanuni Training and Research Hospital \\ Trabzon, Turkey \\ ${ }^{4}$ Department of Neurosurgery \\ Gülhane Military Medical Academy \\ Objective. To describe a rare case of cleidocranial dysplasia, an auto- \\ somal dominant inherited disease involving the skeleton and teeth, \\ with delayed diagnosis. Case report. We report a 24-year-old man \\ with cleidocranial dysplasia admitted with hearing loss, rhinolalia, \\ dyspnea and fatigue. Partial absence of clavicles, a bell-shaped rib- \\ cage, an open frontal fontanel, unerupted permanent teeth and broad \\ sutures were identified at radiographic examination. Conclusion. \\ Cleidocranial dysplasia is very rare, and is commonly missed or diag- \\ nosed late. Radiographic findings are essential for diagnosis. An open \\ frontal fontanel is a particularly important finding for neurosurgeons \\ in diagnosis. We describe this rare case and discuss the clinical fea- \\ tures of CCD.
}

Ankara, Turkey

${ }^{5}$ Department of Pediatric Neurology

Hacettepe University, School of Medicine

Ankara, Turkey

Corresponding author:

Erhan Arslan

Department of Neurosurgery

Giresun University

School of Medicine

Mumcular Sok. N: 1/1

Merkez PC: 28100 Giresun, Turkey

arserhan@gmail.com

Tel.: + 904543101629

Fax.: + 904543101696

Received: 25 June 2013

Accepted: 27 December 2013

Copyright (C) 2014 by

Academy of Sciences and Arts

of Bosnia and Herzegovina.

E-mail for permission to publish:

amabih@anubih.ba
Key words: Open frontal fontanel, Marie and Sainton's disease, Cleidocranial dysplasia, Autosomal dominant.

\section{Introduction}

Cleidocranial dysplasia (CCD) is an autosomal dominant inherited disease (1) caused by mutations in the RUNX2 gene on chromosome 6 p21 encoding a transcription factor involved in osteoblast differentiation and skeletal morphogenesis. CCD was first described by Pierre Marie and Paul Sainton in 1898 (2) and is also known as Marie and Sainton's disease, mutational dysostosis or cleidocranial dysostosis (3). Major characteristics of CCD are delayed closure (ossification) of the fontanels, premature closing of the coronal suture, a protruding mandible, mid-face retrusion, abnormal dentition, including delayed eruption of secondary dentition, failure of primary teeth eruption, supernumerary teeth with dental crowding and malocclusion, and clavicular hypoplasia (4). The nasal bridge is broad due to the wide distance between the orbits (hypertelorism), and patients are more likely to have a high-arched or possibly cleft palate, short stature and scoliosis of the spine. Hand abnormalities are brachydac- 
tyly, tapering fingers, and short, broad thumbs (4). Frontal bossing also occurs because of delayed closure of the anterior fontanel and metopic sutures. ENT manifestations include recurrent paranasal sinus and middle ear infections resulting in conductive hearing loss. Although CCD is a disease with autosomal dominant inheritance, spontaneous genetic mutations have also been reported. Many cases are caused by de novo mutation (4). Diagnosis of CCD is based on clinical and radiological findings.

We describe a case of CCD first diagnosed at the age of 24 years. The patient had mild symptoms and therefore may not have presented to medical professionals previously. It is also a fact that the incidence is so low that the diagnosis is often unrecognised, even when a patient does present.

\section{Case Report}

A 24-year-old man was admitted to the neurosurgery clinic for routine health checks before starting military service. He exhibit- ed symptoms of hearing loss in the right ear, rhinolalia, dyspnea and fatigue. He also had a history of recurrent right middle ear infection. Physical examination revealed delayed closure of the frontal fontanel, a protruding mandible, hypertelorism, frontal bossing, a bell shaped rib-cage, deciduous dentition and delayed eruption of permanent teeth, and partial absence of clavicles. The most interesting findings at physical examination were the open frontal fontanel, extensive mobility of the shoulders due to partial absence of clavicles and his bell shaped chest, as observed at chest radiography (Figure 1).

Cranial radiography and cranial CT scans revealed multiple teeth in both the jaw and maxillary sinuses (Figure 2a, and Figure $2 \mathrm{~b}$ ), open skull sutures, delayed closure of the frontal fontanel, multiple wormian bones, especially in the lambdoid sutures (Figure $2 \mathrm{c}$ and Figura 2d), and narrowing of the internal acoustic canal due to thickness of the petrous bone. Otolaryngological examination revealed a submucosal cleft palate and central perforation in the right

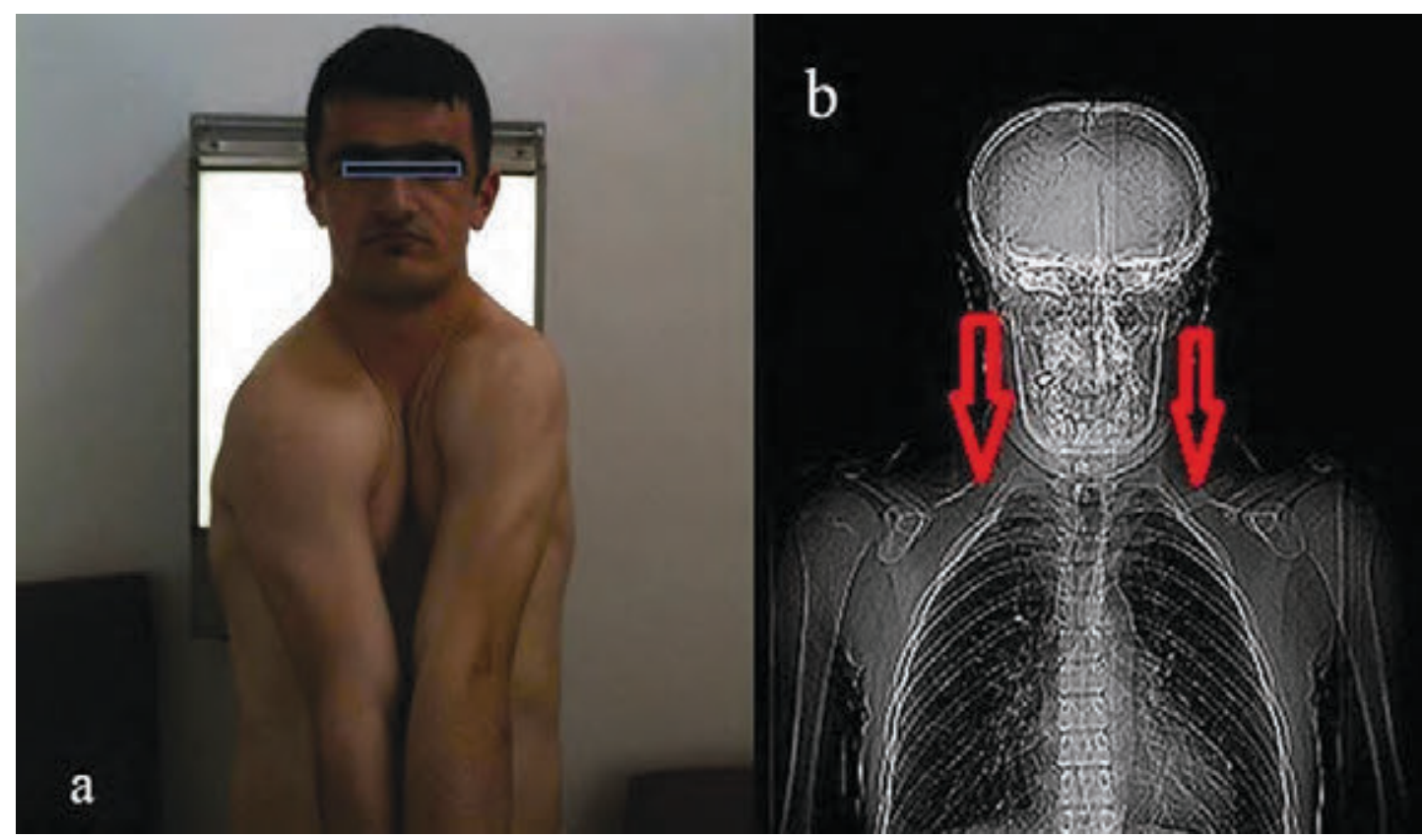

Figure 1 Extensive mobility of shoulders (a) and partial absence of clavicles (red arrows) and bell shaped ribcage on the chest radiography (b). 


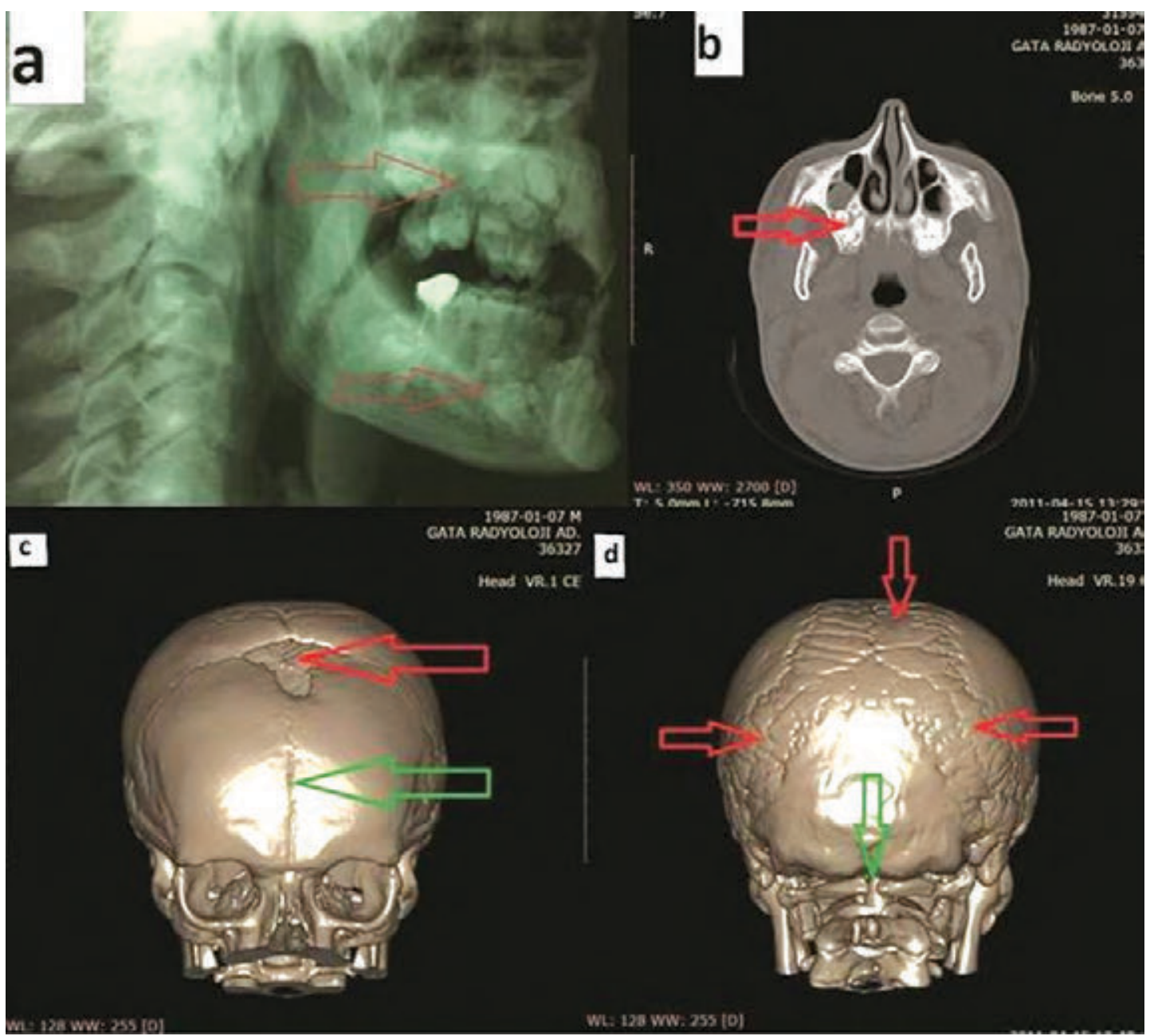

Figure 2 Plain radiographic demonstration of delayed permanent teeth in both jaws (a), and CT scan showing unerupted permanent teeth in the maxilla (b). CT scan showing the delayed closure of the frontal fontanel (red arrow) and frontal groove, resulting from incomplete ossification of metopic suture (green arrow) (c). CT Scan showing the broad sagittal and lambdoid sutures and wormian bones close to lambdoid sutures (red arrows), and open posterior arcus of the axis because of delayed ossification (green arrow) (d).

tympanic membrane. A 40- $\mathrm{dB}$ conductive hearing loss in the right ear was detected at pure-tone audiometry. The patient was diagnosed for the first time with CCD, based on physical examination of the head and oral cavity and X-ray and CT imaging findings.

\section{Discussion}

CCD is a skeletal dysplasia characterized by delayed fontanel closure, hypoplastic or aplastic clavicles and various dental abnormalities (4). Frontal bossing, delayed closure of the frontal fontanel and excessive mobility of the shoulder girdle are the most common and significant findings in CCD ( 1 , 3). Partial or complete absence of clavicles permits the shoulders to be brought forward into close proximity to the chest. Hypertelorism is another common finding associated with frontal bossing (5). This develops as the result of delayed closure of the metopic suture and frontal fontanel (1). The most important and reliable diagnostic method is radiographic evaluation. Radiographic findings of CCD are characteristic and include 
broad cranial sutures, an open frontal fontanel persisting until adulthood, partial or complete absence of the clavicles, numerous wormian bones and large numbers of unerupted supernumerary teeth, bell shaped chest, etc $(1,3)$.

Patients with CCD should be monitored for evidence of conductive or sensorineural hearing loss, which may be due to structural abnormalities of the ossicles, sclerosis of temporal bone together with middle ear problems due to abnormal formation of palate and Eustachian dysfunction, and cochlear or eighth nerve problems (6). Our patient had a submucosal cleft palate, causing rhinolalia, and Eustachian dysfunction resulting in recurrent middle ear infections. The $40-\mathrm{dB}$ conductive hearing loss was due to tympanic membrane perforation, a sequela of recurrent middle ear infections and probably attributable to structural abnormalities of the ossicles or temporal bone sclerosis.

Our patient had also suffered from fatigue and dyspnea. His bell shaped chest was observed on his chest radiography. Decreased respiratory capacity due to abnormal chest shape is the probable cause of fatigue and shortness of breath.

The management strategy for CCD relies on treatment of the manifestations of the syndrome. Dental manifestations can be treated by removal of the supernumerary teeth followed by surgical repositioning of permanent teeth (4). Prosthetic replacements can be used to improve esthetic appearance. Timely treatment of recurrent middle ear infections, and consideration of the possibility of tympanostomy tubes, is necessary to prevent chronic sequelae such as tympanic perforation, adhesive otitis media or conductive hearing loss, as reported in our case. Craniofacial surgery may be required for the correction of skull anomalies (7). Coxa vara is treated with corrective femoral osteotomy. Clavicular fragments can be removed in order to decompress the brachial plexus in cases of symptomatic nerve compression, accompanied by pain and numbness (8). In the case of an open fontanel, appropriate headgear may be recommended for protection against injury. Pregnant women with CCD should be monitored closely for cephalopelvic disproportion. Caesarean section may be the preferred method of delivery in such cases.

CCD can be a challenging syndrome to diagnose and manage, exhibiting multiple developmental deformities of the skeletal system. Early diagnosis is crucial for the timely commencement of appropriate treatment. Successful treatment of patients with CCD requires an interdisciplinary approach and collaboration among specialists, the patient and the family. Even though most cases are diagnosed during childhood or adolescence, diagnosis may be delayed until adulthood if the symptoms and signs are not severe. In our opinion, this report will contribute to the literature and promote awareness of the importance of early diagnosis of this very rare syndrome.

\section{Conclusion}

CCD is a rarely occurring syndrome of skeletal abnormalities, characterized by hypermobility of the shoulders, open fontanels and numerous skeletal findings. Clinical findings are commonly present at birth, but unfortunately diagnosis is not usually made at this early stage. The most specific neurosurgical finding is an open fontanel, and the clinician should be aware of this finding in order to diagnose CCD.

Authors' contributions: Conception and design: CG and EA; Acquisition, analysis and interpretation of data: CG, EA and SP; Revising it critically for important intellectual content: EAA, EA, CG and SA.

Conflict of interest: The authors declare that they have no conflict of interest. 


\section{References}

1. Brueton, Reeve A, Ellis R, Husband P, Thompson EM, Kingston HM. Apparent cleidocranial dysplasia associated with abnormalities of $8 \mathrm{q} 22$ in three individuals. Am J Med Genet. 1992;43(3):612-8.

2. Marie P, Sainton P. Sur la dysostose cleido-cranienne hereditaire. Rev Neurol. 1898;6:835-8.

3. Kallial E, Taskinen PJ. Cleidocranial dysostosis: Report of six typical cases and one atypical case. Oral Surg Oral Med Oral Pathol. 1962;14:808.

4. Mendoza-Londono R, Lee B. Cleidocranial Dysplasia. In: Pagon RA, Adam MP, Bird TD, Dolan CR, Fong CT, Stephens K, editors. GeneReviews ${ }^{\mathrm{Tm}}$ [Internet]. Seattle (WA): University of Washington, Seattle; 1993-2013. [updated 2013 Aug 29].
Available from http://www.ncbi.nlm.nih.gov/ books/NBK1513/PubMed PMID: 20301686.

5. Garg RK, Agrawal P. Clinical spectrum of cleidocranial dysplasia: a case report. Cases J. 2008;1(1):377.

6. Candamourty R, Venkatachalam S, Yuvaraj V, Kumar GS. Cleidocranial dysplasia with hearing loss. J Nat Sci Biol Med. 2013;4(1):245-9.

7. Lovell WW, Winter RB, Morrissy RT, Weinstein SL. Lovell \& Winter's Pediatric Orthopaedics. 6th ed. Philladelphia: Lippincott Williams \& Wilkins; 2006. p. 240.

8. Weinstein SL, Buckwalter JA. Turek's Orthopaedics: Principles and Their Application. 6th ed. Philadelphia: Lippincott Williams \& Wilkins; 2005. p. 251-2. 